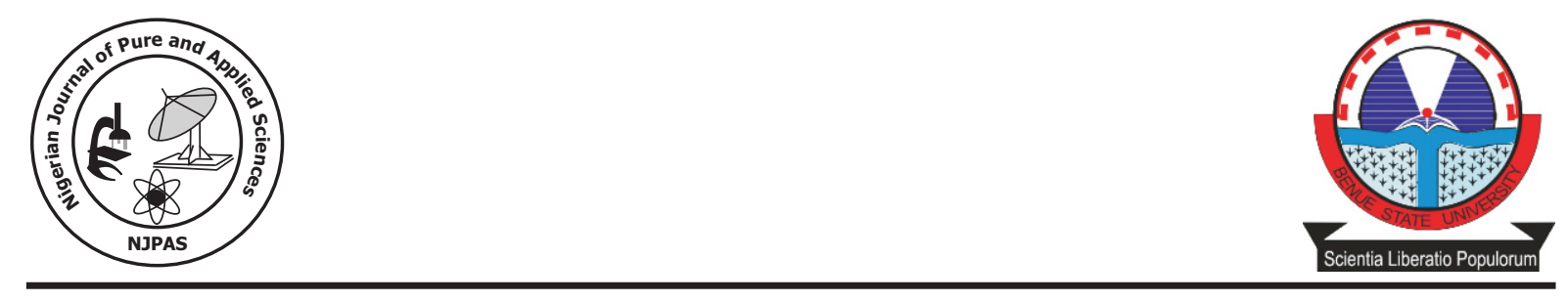

\title{
A Stochastic Model of the Dynamics of Change in Stock Price
}

\author{
Ogwuche, O. I., ${ }^{1}$ Odekunle, M. R. ${ }^{2}$ and Egwurube, M. O. ${ }^{2}$ \\ 1.Department of Mathematics and Computer Science, Benue State University, Makurdi, \\ 2.Department of Mathematics, Federal University of Technology, Yola
}

\begin{abstract}
The solutions of many mathematical models resulting in stochastic differential equations are based on the assumption that the drift and the volatility coefficients were linear functions of the solutions. We formulated a model whose basic parameters could be derived from observations over discretized time intervals rather than the assumption that the drift and the volatility coefficients were linear functions of the solutions. We took into consideration the possibility of an asset gaining, losing or stable in a small interval of time instead of the assumption of the Binomial Asset pricing models that posited that the price could appreciate by a factor $p$ or depreciate by a factor 1-p. A multi-dimensional stochastic differential equation was obtained whose drift is the expectation vector and the volatility the covariance of the stocks with respect to each other. The resulting system of stochastic differential equations was solved numerically using the Euler Maruyama Scheme for multi-dimensional stochastic differential equations through the use of a computer program written in MatLab. We obtained a realization of the evolutions of their prices over a chosen interval of time.
\end{abstract}

\section{Introduction}

The theory of stochastic differential equations has been a subject of study for a long time now. Complicated finite dimensional stochastic dynamics can now be modelled and understood through the use of stochastic calculus and the theory of submartingales (Kloenden and Platen, 1992). Today stochastic differential equations has found application in diverse fields of human endeavour such as finance, civil and mechanical engineering, economics and environmental sciences, chemistry and physics, signal processing and filtering, population dynamics and psychology, pharmacology and medicine to mention just a few. The role of SDE in modelling continuous time stochastic dynamics can be compared to the roles of deterministic ordinary differential equations (ODE) in non random differentiable dynamics.

Stochastic differential equation arises when a deterministic differential equation is randomly perturbed by the white noise and it is characterized by its drift coefficient and the diffusion (volatility) coefficient (Oksendal, 2000). An SDE is therefore a degenerate form of the conventional deterministic differential equations in which the volatility coefficient is zero. A typical SDE can be written as:

$d X_{t}=\mu\left(t, X_{t}\right)+\sigma\left(t, X_{t}\right) d W_{t}$

This can be written in integral form as:

$X_{t}=X_{t_{0}}+\int_{t_{0}}^{t} \mu\left(s, X_{s}\right) d t+\int_{t_{0}}^{t} \sigma\left(s, X_{s}\right) d W_{s}$ 
The first integral in (2) above is the conventional Riemann integral and hence the normal rule of calculus applies. The second integral however is not and hence requires some other rules of integration to be applied. We cannot interpret the second integral as a Riemann integral because it involves integration with respect to a Wienner process which is nowhere differentiable. Further still, it cannot be interpreted a Lebesque or Riemann integral because the Wienner process is not of any known bounded variation (Kloenden and Platen, 1992). Consequently, a new calculus called stochastic calculus was introduced.

Stochastic calculus is a branch of mathematics that operates on stochastic processes. It allows a consistent theory of integration to be defined for integrals of stochastic processes with respect to stochastic processes. It is used to model systems that behave randomly the best known of which is the Wiener process (named in honour of Norbert Wiener), which is used for modelling Brownian motion as described by Albert Einstein and other physical diffusion processes in space of particles subject to random forces. Since the 1970 s, the Wiener process has been widely applied in financial mathematics and economics to model the evolution in time of stock prices and bond interest rates (Hui-Hsiung, 2000).

The Itō calculus named after Kiyoshi Itō extends the methods of calculus to stochastic processes such as Wiener process. It has important applications in mathematical finance and stochastic differential equations. The central concept is the Itō stochastic integral which is a generalization of the ordinary concept of a Riemann-Stieltjes integral. The generalization is in two respects. Firstly, if deals with random variables (more precisely, stochastic processes). Secondly, the method allows integration with respect to a non-differentiable function (technically, stochastic process). An alternative method to the Ito calculus is the Stratonovich calculus which was introduced by Ruslan L. Stratonovich and D.L. Fisk is the preferred method for modelling stochastic processes in applied mathematics (Kloenden and Platen, 1992).

\section{The Ito Formula}

The main tool for solving stochastic differential equations is the Ito formula. Consider a stochastic process $f$. Let $f \in H_{S P}$, that is $\boldsymbol{H}$ is a stochastic process satisfying the following conditions:

I. $f(\alpha) \in H_{R V}$, a random variable. Hence, $\|f(\alpha)\|_{R V}^{2}=E \mid\left(\left.f(\alpha)\right|^{2} \leq k_{1}\right.$ where $k_{1}>0$.

$$
\begin{aligned}
& \text { ii. }\left\|f\left(t_{2}\right)-f\left(t_{1}\right)\right\|_{R V}^{2}=E \mid\left(f\left(t_{2}\right)-\left.f\left(t_{1}\right)\right|^{2} \leq k_{1}\left|t_{2}-t_{1}\right|\right. \\
& \quad \text { for any } t_{2}, t_{1} \in[a, b] \text { for } k_{2}>0
\end{aligned}
$$

iii. $f$ is non anticipating on $[a, b]$

iv. Furthermore let $G:[a, b] X R \rightarrow R$. There exist $k_{3}>0$ such that for $t_{2}, t_{1} \in[a, b]$ and any $X \in H_{S P}$ then $E \mid G\left(t_{2},\left.X\left(t_{2}\right)\right|^{2} \leq k_{3}\right.$ $\left(\left|t_{2}-t_{1}\right|+E \mid X\left(t_{2}\right)-X\left(\left.t_{1}\right|^{2}\right)\right.$

v. For the function $G:[a, b] X R \rightarrow R, G$ $(a, X(a)) \in H_{R V}$ if $X(a) \in H_{R V}$.

\section{Theorem (Ito Formula)}

Let $X \in H_{S P} \quad$ be a stochastic process and let f and $g$ be functions satisfying conditions (I) (iii) above and $\left\|f^{2}(t)\right\|_{R V},\left\|g^{2}(t)\right\|_{R V} \leq k_{4}$

for $t \in[a, b]$. Let $\boldsymbol{F}$ be a function of $\boldsymbol{t}$ and $\boldsymbol{x}$ and let $\boldsymbol{F}(\boldsymbol{t}, \boldsymbol{x})$ have continuous derivative.

$$
\frac{\partial F(t, x)}{\partial t}, \frac{\partial F(t, x)}{\partial x}, \frac{\partial^{2} F(t, x)}{\partial t^{2}}, \frac{\partial^{2} F(t, x)}{\partial x^{2}}, \frac{\partial^{2} F(t, x)}{\partial x \partial t}
$$

for $t \in[a, b]$ and $x \in R$ such that these derivatives satisfies condition (iv) and (v) above. Suppose also that the function

$f(t) \frac{\partial F(t, x)}{\partial x}, \frac{1}{2} g^{2} \frac{\partial^{2} F(t, x)}{\partial x^{2}}, g(t) \frac{\partial F(t, x)}{\partial t}$

satisfy the condition (iv) and (v). Let

$f(t, x)=\frac{\partial F(t, x)}{\partial t}+f(t) \frac{\partial F(t, x)}{\partial x}+\frac{1}{2} g^{2} \frac{\partial^{2} F(t, x)}{\partial x^{2}}$

and $g(t, x)=g(t) \frac{\partial F(t, x)}{\partial t}$

Then, $\mathrm{F}$ satisfies the stochastic differential equation 


$$
d F(t, X(t))=\dot{f}(t, X(t) d t+\dot{g}(t, X(t)) d W(t)
$$

The proof of the theorem is available in literature such as [1], [2], [5] and several others.

\section{Multidimensional Ito formula}

The stock price model developed for the three stocks resulted in a three 3-deimentional system of stochastic differential equations. Hence the tool for solving this system of linear SDE is the multidimensional Ito formula.

Consider the stochastic differential equation and $\boldsymbol{G}(t, \omega)_{i, j}=g_{1}(t, \omega)$ where $\boldsymbol{G}(t, \omega)$ is an $\boldsymbol{n x m}$ matrix. $\boldsymbol{W}(\boldsymbol{t})$ is an $m$ dimensional Wiener process where the elements $W_{i}(t)$ and $W_{i}(t)$ are independent of each other for $i \neq j$. Equation (4) can therefore be written as

$$
d X_{t}(t, \omega)=f_{i}(t, \omega)+\sum_{j=1}^{m} g_{i, j}(t, \omega), \quad i=1,2, \ldots n
$$

This can be written in integral form as $X_{t}(t, \omega)=X_{i}$

$$
(a, \omega)+\int_{a}^{t} f_{i}(s, \omega) d s+\int_{a}^{t} \sum_{j=1}^{m} g_{i, j}(t, \omega) d W_{j}(s, \omega)
$$

The multidimensional Ito formula can be stated as follows:

Let $\boldsymbol{F}(\boldsymbol{t}, \boldsymbol{X})$ be a smooth function of $t$ and $\mathrm{X}$. That is

$F:[a, b] X H_{S P}^{n} \rightarrow R$. Then the Ito formula can be generalized for the multidimensional case as $d F(t, X)$ $=\left(\frac{\partial F}{\partial t}+\sum_{i=1}^{n} \frac{\partial F}{\partial X_{i}} f_{i}+\sum_{i=1}^{n} \sum_{j=1}^{n} \sum_{k=1}^{m} \frac{1}{2} \frac{\partial^{2} F}{\partial X_{i} \partial X_{j}} g_{i, j} g_{j . k}\right)$

$$
d t+\sum_{i=1}^{n} \sum_{j=1}^{m} \frac{\partial F}{\partial x_{i}} g_{i, j} d W_{j}(t)
$$

The theorem and the proof of the Ito formula for the multidimensional case can found in literature such as
[3], [5], [9][11].

\section{Statement of Problem}

Many stochastic models of change in stock price were based on the hypothesis that the drift and diffusion coefficients of the stochastic differential equation are linear functions of the solutions. Then assuming data is available, statistical estimation methods such as the parametric and non-parametric methods will then be used to obtain values for the unknown parameters (Jarrow, et al.,1997). However, if the parameters are derived from basic assumptions, a better understanding of the parameters in the model will be achieved.

Furthermore, the Binomial Asset Pricing model considered the asset price to either go up by a factor $p$ or down by a factor $1-p$. The possibility of an asset price to remain stable over a time interval was not taken into consideration. The focus of this paper is to formulate a stochastic model for stochastic dynamical systems such as stocks whose parameters are derived from some basic assumptions.

\section{Model Formulation}

Consider portfolio of three stocks $S_{1} S_{2 \text {, and }} S_{3}$ we assume that in a small interval of time a $\Delta \mathbf{t}$, stock price may change by losing one unit (-1), remain stable $(0)$ or gain one unit $(+1)$ which we represent by [-1 01 1]. Figure 1 below shows the dynamics of change in the prices of the three stocks.

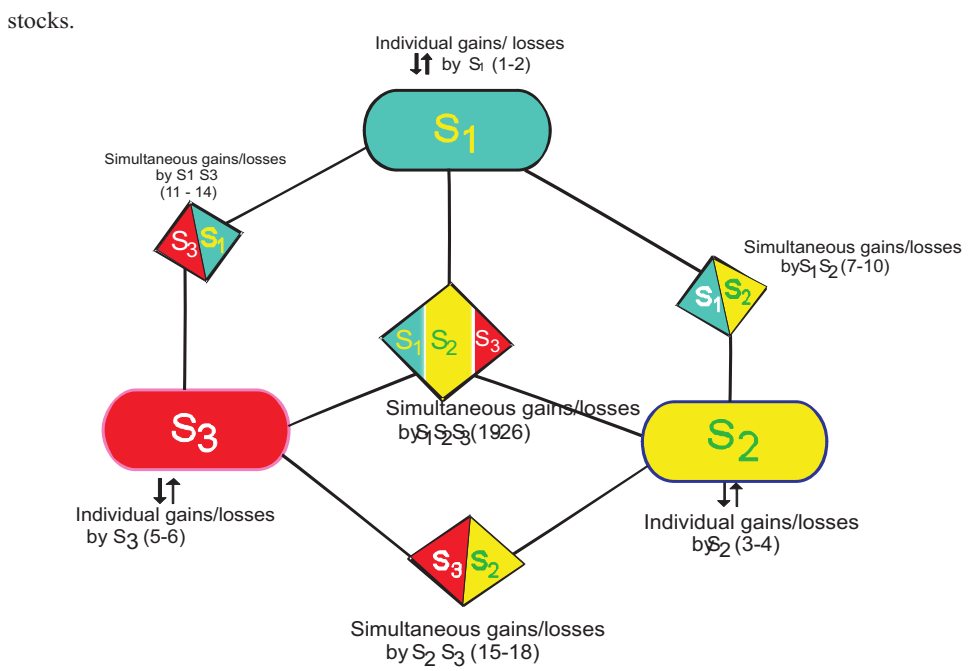

Figure 3.1: Schematic Diagram for the Stochastic Model for Change in Stock Price 
Since there are three stocks and each of them can change in three ways i.e. $-1,0$, or 1 in possibilities. These possibilities are indicated the small interval of time $\Delta \mathbf{t}$, there are $3^{3}=27$ in Table 1 below.

Table 1: Possible outcome of change in 3 stock price and the probabilities

\begin{tabular}{|c|c|c|}
\hline $\mathrm{S} / \mathrm{N}$ & $\begin{array}{l}\text { Change in Stock price } \\
\Delta S=\left[\Delta S_{1}, \Delta S_{2}, \Delta S_{3}\right]^{T}\end{array}$ & Probabilities \\
\hline 1 & {$\left[\begin{array}{lll}-1 & 0 & 0\end{array}\right]$} & $p_{1}=d_{1} S_{1}$ \\
\hline 2 & {$\left[\begin{array}{lll}1 & 0 & 0\end{array}\right]$} & $p_{2}=b_{1} S_{1}$ \\
\hline 3 & {$\left[\begin{array}{lll}0 & 1 & 0\end{array}\right]$} & $p_{3}=b_{2} S_{2}$ \\
\hline 4 & {$\left[\begin{array}{lll}0 & -1 & 0\end{array}\right]$} & $p_{4}=d_{2} S_{2}$ \\
\hline 5 & {$\left[\begin{array}{lll}0 & 0 & -1\end{array}\right]$} & $p_{5}=d_{3} S_{3}$ \\
\hline 6 & {$\left[\begin{array}{lll}0 & 0 & 1\end{array}\right]$} & $p_{6}=b_{3} S_{3}$ \\
\hline 7 & {$\left[\begin{array}{lll}-1 & 1 & 0\end{array}\right]$} & $p_{7}=\alpha_{12} S_{1} S_{2}$ \\
\hline 8 & {$\left[\begin{array}{lll}-1 & -1 & 0\end{array}\right]$} & $p_{8}=\alpha_{11} S_{1} S_{2}$ \\
\hline 9 & {$\left[\begin{array}{lll}1 & 1 & 0\end{array}\right]$} & $p_{9}=\alpha_{22} S_{1} S_{2}$ \\
\hline 10 & {$\left[\begin{array}{lll}1 & -1 & 0\end{array}\right]$} & $p_{10}=\alpha_{21} S_{1} S_{2}$ \\
\hline 11 & {$\left[\begin{array}{lll}-1 & 0 & 1\end{array}\right]$} & $p_{11}=\beta_{12} S_{1} S_{3}$ \\
\hline 12 & {$\left[\begin{array}{lll}-1 & 0 & -1\end{array}\right]$} & $p_{12}=\beta_{11} S_{1} S_{3}$ \\
\hline 13 & {$\left[\begin{array}{lll}1 & 0 & 1\end{array}\right]$} & $p_{13}=\beta_{22} S_{1} S_{3}$ \\
\hline 14 & {$\left[\begin{array}{lll}1 & 0 & -1\end{array}\right]$} & $p_{14}=\beta_{21} s_{1} S_{3}$ \\
\hline 15 & {$\left[\begin{array}{lll}0 & 1 & 1\end{array}\right]$} & $p_{15}=\gamma_{22} S_{2} S_{3}$ \\
\hline 16 & {$\left[\begin{array}{lll}0 & -1 & -1\end{array}\right]$} & $p_{16}=\gamma_{11} s_{2} S_{3}$ \\
\hline 17 & {$\left[\begin{array}{lll}0 & -1 & 1\end{array}\right]$} & $p_{17}=\gamma_{12} S_{2} S_{3}$ \\
\hline 18 & {$\left[\begin{array}{lll}0 & 1 & -1\end{array}\right]$} & $p_{18}=\gamma_{21} S_{2} S_{3}$ \\
\hline 19 & {$\left[\begin{array}{lll}-1 & 1 & 1\end{array}\right]$} & $p_{19}=\eta_{122} S_{1} S_{2} S_{3}$ \\
\hline 20 & {$\left[\begin{array}{lll}-1 & 1 & -1\end{array}\right]$} & $p_{20}=\eta_{121} s_{1} s_{2} s_{3}$ \\
\hline 21 & {$\left[\begin{array}{lll}-1 & -1 & -1\end{array}\right]$} & $p_{21}=\eta_{111} s_{1} s_{2} s_{3}$ \\
\hline 22 & {$\left[\begin{array}{lll}-1 & -1 & 1\end{array}\right]$} & $p_{22}=\eta_{112} S_{1} S_{2} S_{3}$ \\
\hline 23 & {$\left[\begin{array}{lll}1 & -1 & -1\end{array}\right]$} & $p_{23}=\eta_{211} S_{1} S_{2} S_{3}$ \\
\hline 24 & {$\left[\begin{array}{lll}1 & 1 & -1\end{array}\right]$} & $p_{24}=\eta_{221} S_{1} S_{2} S_{3}$ \\
\hline 25 & {$\left[\begin{array}{lll}1 & -1 & 1\end{array}\right]$} & $p_{25}=\eta_{212} S_{1} S_{2} S_{3}$ \\
\hline 26 & {$\left[\begin{array}{lll}1 & 1 & 1\end{array}\right]$} & $p_{26}=\eta_{222} S_{1} S_{2} S_{3}$ \\
\hline 27 & {$\left[\begin{array}{lll}0 & 0 & 0\end{array}\right]$} & $p_{27}=1-\sum^{26} p_{i}$ \\
\hline
\end{tabular}

Here, $\Delta \mathbf{t}$ represent change in stock price. For example, $\Delta \boldsymbol{S}=\left[\begin{array}{lll}1 & 0 & 0\end{array}\right]$ represent a gain of 1 unit in stock $\boldsymbol{S}_{1}$ while stock $\boldsymbol{S}_{2}$ and $\boldsymbol{S}_{3}$ remain stable; represent a simultaneous gain of 1 unit by stock $\boldsymbol{S}_{1}$ and $\boldsymbol{S}_{3}$ and a loss of 1 unit stock $\boldsymbol{S}_{2}$. It is assumed that the change in the stock price is proportional to the price of the stock. For simultaneous gains/losses, we assume that the probability of the change is proportional to the product of the stock prices. This is reasonable as, supposing that the one of the stock price is zero then, the probability of a simultaneous gain is zero. It is also assumed that $\Delta \mathbf{t}$ is sufficiently small so that $\boldsymbol{P}_{27}$ which is the probability that there is no change in the three stocks prices within the time interval $\Delta \mathbf{t}$ is positive.

The parameters $\boldsymbol{b}_{\boldsymbol{i}}, \boldsymbol{d}_{\boldsymbol{i}} \boldsymbol{i}=\mathbf{1}, \ldots, \mathbf{3}$, defines the rate at which stocks experience individual gains or losses respectively. The parameter

$\alpha_{j, k}, \beta_{j, k}, \gamma_{j, k} \eta_{j, k, l}$ for $j, k, l=1,2$ defines the rate at which stocks experience simultaneous gains and/or losses with each parameter depending on $\boldsymbol{t}$. For example, $b_{i} S_{i} \Delta t$ is the probability that stock i gains one unit in the time interval $\Delta \mathbf{t}$ The change involving $\boldsymbol{S}_{1}$ and $\boldsymbol{S}_{2}$ and $\boldsymbol{S}_{2}$ and $\boldsymbol{S}_{3}$ simultaneously is denoted by $\boldsymbol{\alpha}_{j, k}$. Also, $\boldsymbol{\beta}_{j, k}, \gamma_{j, k}$ denotes the changes involving $\boldsymbol{S}_{1}$ and $\boldsymbol{S}_{3} \boldsymbol{S}_{2}$ and $\boldsymbol{S}_{3}$ simultaneously. For example, $\boldsymbol{\alpha}_{1,2}$ represent the change involving the two stocks $\boldsymbol{S}_{1} \boldsymbol{S}_{2}$ in which $\boldsymbol{S}_{1}$ gains while $\boldsymbol{S}_{2}$ losses. Also $\beta_{2,1}$ represent the change involving the two stocks $S_{1} S_{3}$ in which $S_{1}$ losses while gains. Finally, $\eta_{j, k, l}$ denote the change in the three stocks $\boldsymbol{S}_{1}$ and $\boldsymbol{S}_{2}$ and $\boldsymbol{S}_{2}$ simultaneously. In all cases, the subscript 1 or 2 represent loss of one unit or gain of one unit 
respectively. It should be noted that $\sum_{i=1}^{27} p_{i}=1$.

Using the above representations for $\boldsymbol{p}_{\boldsymbol{i}}$ and $\Delta \boldsymbol{S} \boldsymbol{i}$ the expectation vector is derived as follows:

$$
E(\Delta S)=\sum_{i=1}^{27} p_{i} \Delta S_{i}=\left[\begin{array}{l}
f_{1} \\
f_{2} \\
f_{3}
\end{array}\right]
$$

$f_{1}=\left(d_{1}+b_{1}\right) S_{1}+\left(\alpha_{12}+\alpha_{21}+\alpha_{22}+\alpha_{11}\right)$

$S_{1} S_{2}+\left(\beta_{12}+\beta_{21}+\beta_{22}+\beta_{11}\right) S_{1} S_{3}+\left(\eta_{122}+\right.$

$\eta_{121}+\eta_{111}+\eta_{112}+\eta_{211}+\eta_{221}+\eta_{212}$

$\left.+\eta_{222}\right) S_{1} S_{2} S_{3}$

$f_{1}$ Represent the totality of the likelihood of change in volving stock $\boldsymbol{S}_{\boldsymbol{I}}$

$f_{2}=\left(d_{2}+b_{2}\right) S_{2}+\left(\alpha_{12}+\alpha_{21}+\alpha_{22}+\alpha_{11}\right)$

$S_{1} S_{2}+\left(\gamma_{22}+\gamma_{21}+\gamma_{22}+\gamma_{11}\right) S_{2} S_{3}+\left(\eta_{122}+\right.$

$\eta_{121}+\eta_{111}+\eta_{112}+\eta_{211}+\eta_{221}+\eta_{212}+$

$\left.\eta_{222}\right) S_{1} S_{2} S_{3}$.

$\boldsymbol{f} \boldsymbol{z}$ Represent the totality of the likelihood of change involving stock $\boldsymbol{S}_{2}$

$f_{3}=\left(d_{3}+b_{3}\right) S_{3}+\left(\beta_{22}+\beta_{11}+\beta_{22}+\beta_{11}\right)$

$S_{1} S_{3}+\left(\gamma_{22}+\gamma_{11}+\gamma_{22}+\gamma_{21}\right) S_{2} S_{3}+\left(\eta_{122}+\right.$

$\eta_{121}+\eta_{111}+\eta_{112}+\eta_{211}+\eta_{221}+\eta_{212}+$

$\left.\eta_{222}\right) S_{1} S_{2} S_{3} \cdot(11)$

$f_{3}$ Represent the totality of the likelihood of change involving stock $S_{3}$. Putting

$d S_{1}=\left(d_{1}+b_{1}\right) S_{1}$

$d S_{2}=\left(d_{2}+b_{2}\right) S_{2}$, $d S_{3}=\left(d_{3}+b_{3}\right) S_{3}$,

$d S_{1} S_{2}=\left(\alpha_{12}+\alpha_{21}+\alpha_{22}+\alpha_{11}\right) S_{1} S_{2}$,

$d S_{1} S_{3}=\left(\beta_{12}+\beta_{21}+\beta_{22}+\beta_{11}\right) S_{1} S_{3}$,

$d S_{2} S_{3}=\left(\gamma_{12}+\gamma_{21}+\gamma_{22}+\gamma_{11}\right) S_{2} S_{3}$.

$d S_{1} S_{2} S_{3}=\left(\eta_{122}+\eta_{121}+\eta_{111}+\eta_{112}+\eta_{211}\right.$

$\left.+\eta_{221}+\eta_{212}+\eta_{222}\right) S_{1} S_{2} S_{3}$

Then the covariance matrix is derived as follows:

$E(\Delta S(\Delta S))^{T}=\left[\begin{array}{ccc}d S_{1} & d S_{1} S_{2} & d S_{1} S_{3} \\ d S_{2} S_{1} & d S_{2} & d S_{2} S_{3} \\ d S_{3} S_{1} & d S_{3} S_{2} & d S_{3}\end{array}\right]$

Clearly, the covariance matrix is a positive symmetric and hence has a positive square root.

Denoting $B=(V)^{\frac{1}{2}}$. As $\Delta t \rightarrow 0$, the probability distribution of the stock price approximates the probability distribution of the solution of the system of stochastic differential equation [3]

$d S(t)=\mu\left(t, S, S_{2}, S_{3}\right) d t+B\left(t, S, S_{2}, S_{3}\right)$

$d W(t)$

where

$\mu\left(t, S, S_{2}, S_{3}\right)=\left[\begin{array}{l}f_{1} \\ f_{2} \\ f_{3}\end{array}\right]$

and

$B\left(t, S_{1}, S_{2}, S_{3}\right)=\left[\begin{array}{ccc}d S_{1} & d S_{1} S_{2} & d S_{1} S_{3} \\ d S_{2} S_{1} & d S_{2} & d S_{2} S_{3} \\ d S_{3} S_{1} & d S_{3} S_{2} & d S_{3}\end{array}\right]^{1 / 2}$

Numerical Experiments

To test the model, random probabilities were assigned for each possible change in the price of the stocks 
Table 2: Simulated Probabilities of events

\begin{tabular}{|c|c|c|}
\hline $\mathrm{S} / \mathrm{N}$ & $\begin{array}{l}\text { Change in Stock price } \\
\Delta S=\left[\Delta S_{1}, \Delta S_{2}, \Delta S_{3}\right]^{T}\end{array}$ & Probabilities \\
\hline 1 & {$\left[\begin{array}{lll}-1 & 0 & 0\end{array}\right]$} & 0.047 \\
\hline 2 & {$\left[\begin{array}{lll}1 & 0 & 0\end{array}\right]$} & 0.052 \\
\hline 3 & {$\left[\begin{array}{lll}0 & 1 & 0\end{array}\right]$} & 0.007 \\
\hline 4 & {$\left[\begin{array}{lll}0 & -1 & 0\end{array}\right]$} & 0.052 \\
\hline 5 & {$\left[\begin{array}{lll}0 & 0 & -1\end{array}\right]$} & 0.036 \\
\hline 6 & {$\left[\begin{array}{lll}0 & 0 & 1\end{array}\right]$} & 0.006 \\
\hline 7 & {$\left[\begin{array}{lll}-1 & 1 & 0\end{array}\right]$} & 0.046 \\
\hline 8 & {$\left[\begin{array}{lll}-1 & -1 & 0\end{array}\right]$} & 0.031 \\
\hline 9 & {$\left[\begin{array}{lll}1 & 1 & 0\end{array}\right]$} & 0.055 \\
\hline 10 & {$\left[\begin{array}{lll}1 & -1 & 0\end{array}\right]$} & 0.055 \\
\hline 11 & {$\left[\begin{array}{lll}-1 & 0 & 1\end{array}\right]$} & 0.009 \\
\hline 12 & {$\left[\begin{array}{lll}-1 & 0 & -1\end{array}\right]$} & 0.055 \\
\hline 13 & {$\left[\begin{array}{lll}1 & 0 & 1\end{array}\right]$} & 0.055 \\
\hline 14 & {$\left[\begin{array}{lll}1 & 0 & -1\end{array}\right]$} & 0.027 \\
\hline 15 & {$\left[\begin{array}{lll}0 & 1 & 1\end{array}\right]$} & 0.046 \\
\hline 16 & {$\left[\begin{array}{lll}0 & -1 & -1\end{array}\right]$} & 0.008 \\
\hline 17 & {$\left[\begin{array}{lll}0 & -1 & 1\end{array}\right]$} & 0.024 \\
\hline 18 & {$\left[\begin{array}{lll}0 & 1 & -1\end{array}\right]$} & 0.052 \\
\hline 19 & {$\left[\begin{array}{lll}-1 & 1 & 1\end{array}\right]$} & 0.045 \\
\hline 20 & {$\left[\begin{array}{lll}-1 & 1 & -1\end{array}\right]$} & 0.055 \\
\hline 21 & {$\left[\begin{array}{lll}-1 & -1 & -1\end{array}\right]$} & 0.038 \\
\hline 22 & {$\left[\begin{array}{lll}-1 & -1 & 1\end{array}\right]$} & 0.002 \\
\hline 23 & {$\left[\begin{array}{lll}1 & -1 & -1\end{array}\right]$} & 0.049 \\
\hline 24 & {$\left[\begin{array}{lll}1 & 1 & -1\end{array}\right]$} & 0.053 \\
\hline 25 & {$\left[\begin{array}{lll}1 & -1 & 1\end{array}\right]$} & 0.039 \\
\hline 26 & {$\left[\begin{array}{lll}1 & 1 & 1\end{array}\right]$} & 0.043 \\
\hline 27 & {$\left[\begin{array}{ll}0 & 0\end{array}\right]$} & 0.043 \\
\hline
\end{tabular}

Using the values in the table above, and were calculated accordingly as:

$\mathrm{f} 1=7990 ; \mathbf{f} 2=0.7430 ; \mathrm{f3}=0.6850$. Hence, the expectation vector is:

$E(\Delta S)=\sum_{i=1}^{27} P \Delta S=\left(\begin{array}{l}f_{1} \\ f_{2} \\ f_{3}\end{array}\right)=\left[\begin{array}{l}0.7990 \\ 0.7430 \\ 0.6850\end{array}\right]$

and the covariance matrix

$E(\Delta S(\Delta S))^{T}=\left[\begin{array}{ccc}\Delta S & \Delta S_{1} S_{2} & \Delta S_{1} S_{3} \\ \Delta S_{2} S_{1} & \Delta S_{2} & \Delta S_{2} S_{3} \\ \Delta S_{3} S_{1} & \Delta S_{3} S_{2} & \Delta S_{3}\end{array}\right]$

$$
=\left[\begin{array}{ccc}
0.0990 & 0.1870 & 0.1460 \\
0.1870 & 0.0590 & 0.1300 \\
0.1460 & 0.1300 & 0.3670
\end{array}\right]
$$

This give rise to the SDE $d S_{t}=\mu\left(t, S_{1}, S_{2}, S_{3}\right) d t+B\left(t, S_{1}, S_{2}, S_{3}\right) d W_{t}$ where $d S_{t}=\mu\left(t, S_{1}, S_{2}, S_{3}\right)=\left[\begin{array}{l}0.7990 \\ 0.7430 \\ 0.6850\end{array}\right]$ and

$$
B\left(t, S_{1}, S_{2}, S_{3}\right)=\left[\begin{array}{ccc}
0.0990 & 0.1870 & 0.1460 \\
0.1870 & 0.0590 & 0.1300 \\
0.1460 & 0.1300 & 0.3670
\end{array}\right] .
$$

\section{Numerical Solution}

It is well known that only few stochastic differential equations can be solved analytically. We therefore solved the resulting system of stochastic differential equations above using the Euler- Maruyama scheme for the multidimensional case which is well discussed in [6], [7]. The scheme is give as: $S_{n+1}^{k}=S_{n}^{k}+\mu^{k} \Delta t+\sum_{j=1}^{k} B^{k, j} d W_{t}^{k}$.

Simulations of the trajectories using the time interval of 0.005 for 360 times were as shown below in figure 1-4 

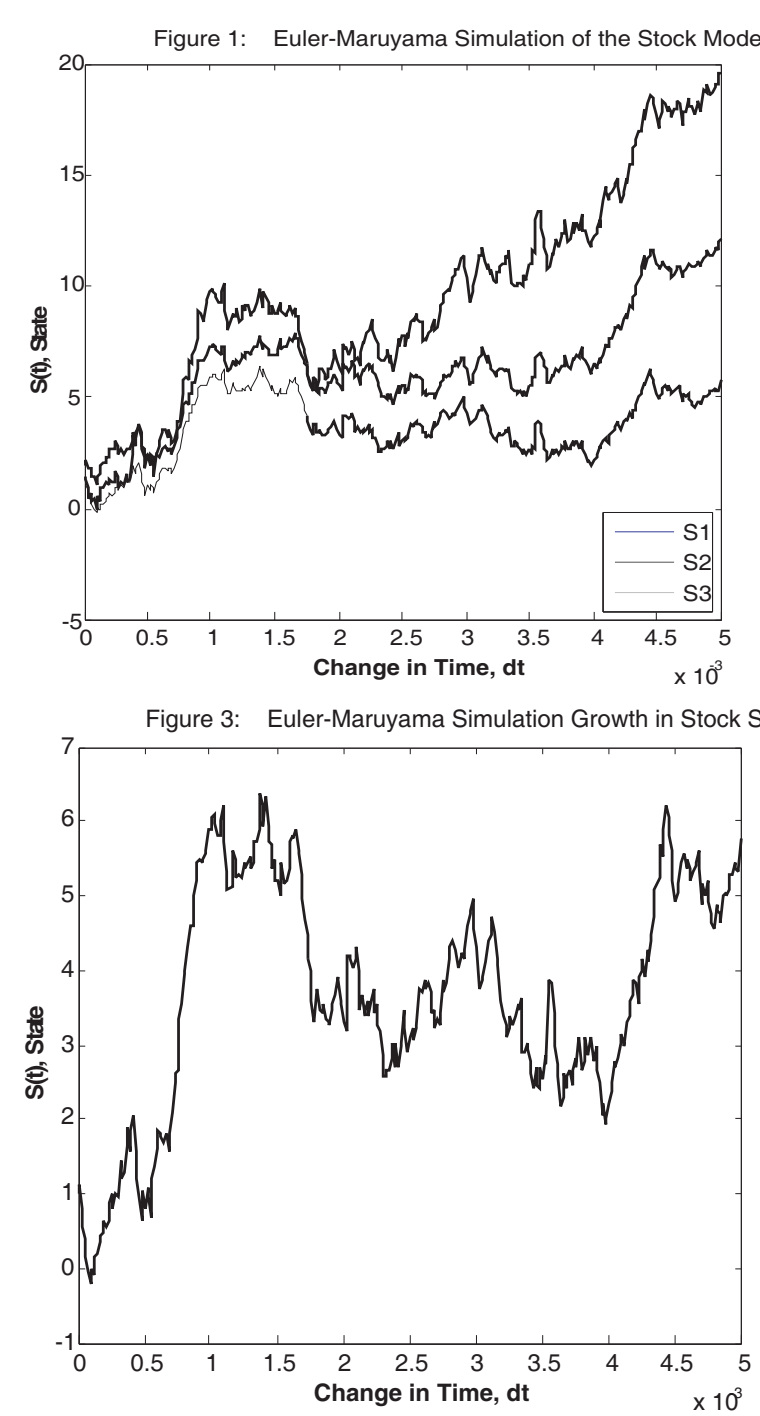

\section{References}

Allen E. (2007), Modeling with Ito Stochastic Differential Equations, Springer Dodrecht, the Netherlands

Hui-Hsiung K. (2000), Introduction to Stochastic Integration, Springer, USA.

Jarrow, R., Lando D. \& Turnbull S. (1997), A Markov model for the term structure of credit risk spreads, Review, Financial Studies, 10(2), 481-523

Jorion, P. (1988). On jump process in the foreign exchange and stock markets, A Review,

Klebaner F.C. (2005), Introduction to Stochastic Calculus with Applications, Imperial Press, London.
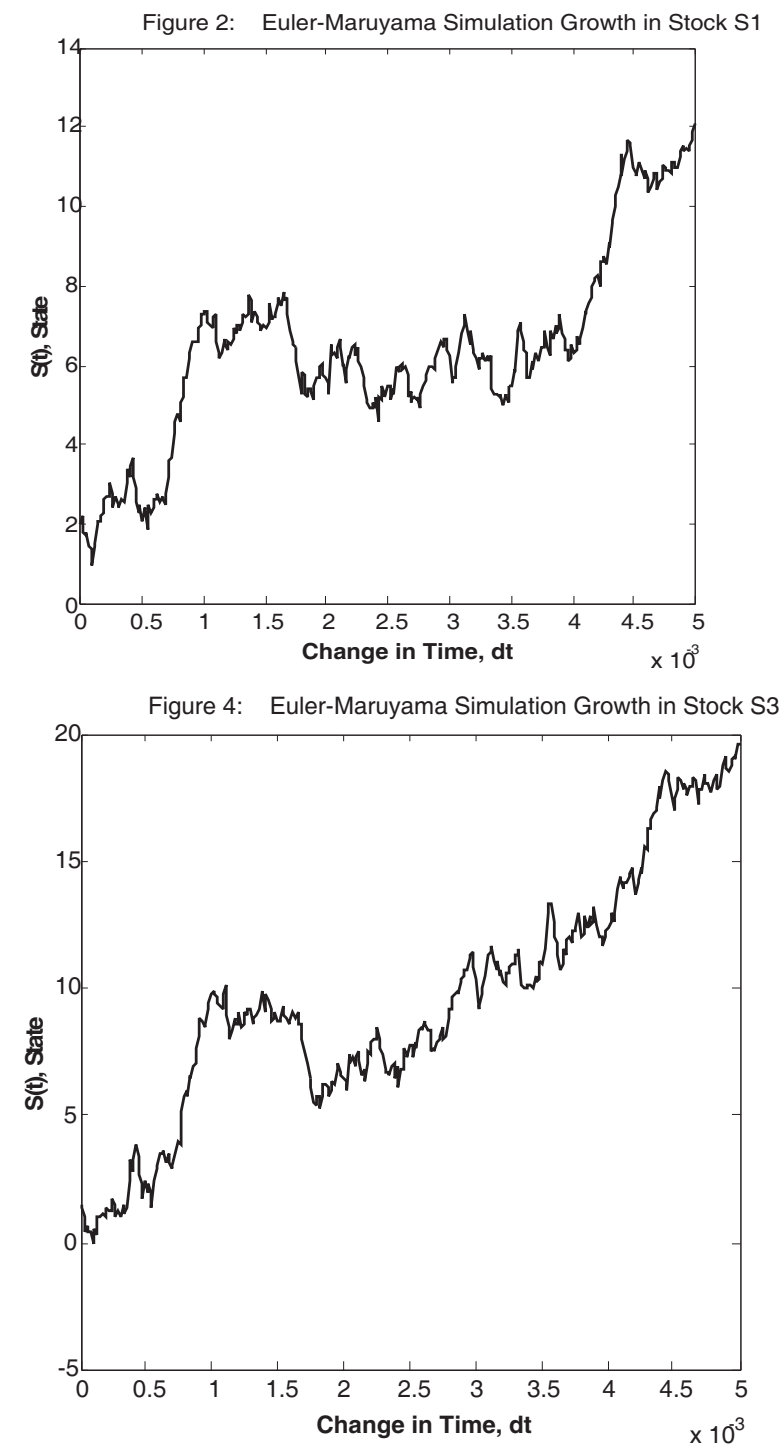

Kloeden P.E., Platen E. (1992), Numerical Solutions of Stochastic Differential Equations, Springer, New York

Kloeden P.E., Platen E. and Schurz H. 1994), Numerical Solutions of SDE through Computer Experiment, Springer, New York

Kuo, H. (2000), Introduction to Stochastic Integration, Springer, USA

Oksendal B.C. (2000). Stochastic Differential Equations, An introduction with Applications, Sixth Edition, Springer, New York.

Platen, E, Nicola Bruti-Liberati (2010), Numerical Solutions of Stochastic Differential Equations in Finance, Springer, New York

Sheldon L (2006), Introductory Stochastic Analysis for Finance and Insurance, John Wiley \& Sons, New Jessy 